\title{
Home Education for Children with Newly Diagnosed Type-1 Diabetes-Exploring Needs and Experiences among Participants of Home Education Visits
}

Truelsen MF'*, Christiansen SY2 ${ }^{2}$ LundbyChristensen $\mathrm{L}^{2,3}$ and Lehn $\mathbf{S F}^{2}$

${ }^{1}$ Department of Pediatrics, Holbaek Hospital, Denmark ${ }^{2}$ Steno Diabetes Center Zealand, Zealand, Denmark ${ }^{3}$ Department of Pediatric and Adolescent Medicine, Naestved-Slagelse-Ringsted Hospitals, Denmark

*Corresponding author: Truelsen MF, Department of Pediatrics, Holbaek Hospital, Gl. Klausdalsbrovej 475B, DK-2730 Herlev, Denmark

Received: J une 30, 2021; Accepted: July 19, 2021; Published: J uly 26, 2021

\begin{abstract}
Objective: Type 1-diabetes is increasing among younger age groups and type 1-diabetes has a major impact on daily life of both the children and their families. The aim of the current study was to explore a family and network centered intervention to children with newly diagnosed type-1 diabetes. Home education visits performed by health care professionals were evaluated using focus group interviews.
\end{abstract}

Method: We conducted five focus group interviews with 21 home education attendants and two individual interviews with health care professionals. Employing inductive content analysis.

Results: Families of children who have newly been diagnosed with type 1-diabetes struggle to comply with diabetes care and, thus, leaving the children to the care of others is a major concern. Lack of knowledge among members of family and social network, adhere to an anxiety of doing something wrong in relation to diabetes care. Home education visits educate the wider family and social network about diabetes care. Focus group participants and health professionals describe that the education visits constitute a major support for parents in the task of instructing family and social network members. Additionally, family and social network members report that they feel more confident to contribute more to the care of the child with type 1-diabetes.

Conclusion: Existing prejudices and lack of knowledge leads to stress and worry among the social network of children with type-1 diabetes. Home education improves knowledge and feeling of confidence concerning diabetes care among family and social network, with the potential to strengthen and maintain close social relations.

Keywords: Type 1 diabetes; Education; Home education visit; Family centered; Social network; Children

\section{Abbreviations \\ T1D: Type-1 Diabetes \\ Introduction}

Type-1 Diabetes (T1D) is one of the most common chronic diseases to appear in childhood and the incidence is increasing. In Denmark, the incidence has been increasing in the younger age groups for more than 20 years [1]. According to the International Society for Pediatric and Adolescent Diabetes, the overall goal and purpose of diabetes treatment is "to provide care that results in high quality of life, normal growth and development, and lowest attainable risk of acute and long-term diabetes complications" [2]. Nevertheless, when a child is diagnosed with T1D the whole family is faced with chronic disruption $[3,4]$ and increased risk of poor mental health $[5,6]$. The onset of T1D has numerous consequences for family life. The majority of parents experience chronic stressors such as fear of hypoglycemia $[7,8]$. Treatment of T1D is complex and families must acquire extensive knowledge on self-care. Children with T1D need monitoring of blood glucose and daily insulin treatment corresponding to the intake of carbohydrates and physical activity. The child requires continuous support from parents and other family members. Once families have adapted to the new situation following T1D diagnosis, they describe the urge to 'get back to normal' and e.g. letting the child stay in the care of others for short durations of time [6]. Yet, handing over care of a child with T1D to others is a major challenge for most parents. The extended family network is not routinely offered training to provide care for the child. Consequently, the families often have a poor caregiver network to rely on to be supportive in times of need. A recent social network factor study highlights the importance of social network in families with adolescents with T1D. Social network is continuously a source of tangible and informational support and provide help with daily disease management and is crucial for family resilience [9].

Home-based care, where health care professionals visit the family in their home, has the potential of improving the education and skills among the child's supporting network [10]. However, studies evaluating the benefits and barriers of home education are sparse and more studies are needed to illuminate the potential outcome [11-14]. 
Home education visits to families and network of a child with newly diagnosed T1D are not standard care in pediatric outpatient clinics in Denmark. However, this study explore home education visits as a novel practice engaged by a pediatric outpatient clinic in a hospital setting in the Eastern Denmark. We aim to explore the perceived need for and experience with home education visits among families and the social network of children with T1D who have recently participated in home education visits. Further, we explore the experiences and perspectives of the health care professionals performing home education visits.

\section{Material and Methods}

The study reporting is informed by the Consolidated Criteria for Reporting Qualitative Studies [15].

\section{Home education visits}

Home education visits are not part of standard care in pediatric outpatient clinics in Denmark. This qualitative study explores home education visits which extraordinarily have been performed by a pediatric outpatient clinic at a Danish hospital since 2013. The pediatric outpatient clinic offers all families, of a child or adolescent with newly diagnosed T1D, a home education visit. The visits are performed by a multidisciplinary team consisting of a diabetologist and a dietician. They take place within $4-8$ weeks after diagnosis and lasts about $1 \frac{1}{2}$ to 2 hours. The visits are planned in cooperation with the families, and the families are encouraged to invite all people from their social network who play a role in the everyday life and wellbeing of the child. The home education visits intend to educate the family and social network to ensure extended support for the child and family when it comes to managing T1D.

\section{Study design}

We explored the perceived need for and experiences with home education visits by engaging a qualitative inductive study design. Initially, two of the research group participants (SYC and SFL) made participated in two home education visits each (two hours per visit) as observers. Field observations fed into the design of an interview guide. Subsequently, we performed semi-structured focus group interviews with families and other participants of the home education visits. Focus group interviews were moderated by an experienced pediatric diabetes nurse (MFT) assisted by a health professional with qualitative interview training and experience (SYC). The latter additionally performed individual interviews with two health care professionals who both had six years of experience performing the home education visits in question.

\section{Recruitment and participants}

We recruited participants from five home education visits taking place from May 2019 to January 2020. In order to obtain broad insight into the experiences with educational home visits, we employed a strategy of maximum variation sampling [16]. Hence, we included participants with varying social relations and children with differentiating ages as outlined. To recruit the families, we initially asked them at the outpatient clinic if they would like to be contacted by the research team and possibly participate in interviews. If the families consented, they were contacted by phone by one of the researchers (MFT).
We conducted five focus groups interviews. The children with T1D included one preschool child, three young school children (age six to 12) and one adolescent. Besides the children with T1D, focus groups included parents (all groups), siblings (one group), grandparents (all groups), aunt (one group) and parent of a playmate (one group). In total 21 individuals participated in focus groups. Four interviews were conducted in the homes of the families and one was conducted in the outpatient clinic. All in the time range one to seven month after the home education visit. All participants above 18 years gave written informed consent while participants under the age of 18 provided consent through their parents.

The two health professionals from the pediatric outpatient clinic performing the home education visits were interviewed individually.

\section{Data analysis}

All interviews were audio-recorded and transcribed verbatim and analyzed using content analysis [17]. NVivo, a qualitative software package (QSR International 2020), was used to facilitate data coding using an inductive analysis strategy [17]. We let analytic abstractions arise from the interview data, so the analysis reflected the informants' perceptions as truly as possible [17]. Hence, we analytically followed a path of open coding, grouping, categorizing and abstraction of interview data. In all of these steps participating researchers discussed analytical founds, moving back and forth in the analytical process. Three of the authors (MFT, SYC, SFL) did open coding separately and researchers discussed findings until reaching consensus. Very few discrepancies appeared in this process. Further, the grouping of coding was performed in collaboration (MFT, LLC, SYC and SFL). The researchers shared the process of abstraction and writing among them and all findings were discussed in the author group.

\section{Results}

Below we elaborate on the professional perspective on the purpose and the structure of the home education visits. Further, we unfold some of the major challenges the families experience in the first period after the child is diagnosed with T1D, which also motivated families to host and attend the visits. Subsequently, we describe the perceived value of home education visits. The value of home education visits appears to be different depending on the participants' social role and relation to the child, and the results are therefore divided according to relation. Ultimately, we unfold the premises and limitations of the home education visits.

\section{Purpose and structure of home education visits}

Health care professionals experience that parents of children with T1D face constant worry and a need to pay constant attention to diabetes management, which challenge family life. Grandparents and others from the social network need to support the family and take care of the child occasionally without the parents present. Hence, a central purpose of home education visits is to reduce fears and barriers of support in the social network. Health care professionals intend to defuse worry and anxiety by informing about diabetes and diabetes treatment. The diabetologist describes:

"Most relatives know about hypoglycemia and believe that if their little baby [child with T1D] gets it, they will die - but of course they won't. We have learned that we actually have to say out loud that their children won't die from this." 
Both health care professionals and focus group participants perceive the learning that social network can take care of the child, without fatal consequence, as one of the most important learnings from the home education visits.

Another important objective for the health care professionals is building a closer relationship with the families and gaining more knowledge about their family life and any potential challenges. This provides insight into special needs of the child and if the family will benefit from an intensified level of support.

According to the health care professionals the home education visits teach the participants about diabetes, high and low blood glucose and how to treat it, insulin treatment, and how to count carbohydrates. If possible, they include hands-on practice with the technological devices. However, the health care professionals emphasize that the agenda is open and directed towards the subjects brought to the table by the participants. E.g. with older children, the health care professionals additionally talk about teenage-related issues like how to handle alcohol intake when having diabetes.

\section{Needs and challenges in the first time following T1D diagnosis}

The first period after diagnosis: The parents from the focus group interviews described how they faced several challenges in the first period after diagnosis. The struggle to return to an everyday life with diabetes as an integral part was overwhelming. One of the main tasks was to manage the child's blood glucose levels, via technological equipment such as an insulin pump and a glucose sensor. The families described how this task alone took up a lot of time and resources during the first weeks. Simultaneously they needed to cope with the emotional stress following the fact that they now had a child with a chronic condition.

Parents described how they often felt anxious about leaving their child with other caregivers such as grandparents, schoolteachers etc. Handing over the responsibility for the treatment of T1D was a big issue.

The level of anxiety was depending somewhat on the age of the child. Parents of the younger children described that a few hours during the day was okay, but when it came to an overnight stay, they felt it was too much to ask of others to check their child's blood glucose during the night.

The older children from the focus groups were less dependent on support from caregivers. However, parents underlined the importance of understanding and support from adults surrounding the child regardless of age.

Misconceptions about T1D: The families met prejudices and misconceptions on how to treat and lead a life with T1D often deriving from lack of knowledge, outdated knowledge or confusion between T1D and T2D. Several parents mentioned the common misunderstanding that T1D is a disease triggered by an unhealthy lifestyle and that parents or the child itself could have prevented it. This misconception created a sense of fault on the children and their families. The parents felt it was a burden to defend themselves and correct these misunderstandings.

Parents also reported that they received many questions regarding the child's diet and lifestyle. Some parents felt that people distrusted their answers, especially regarding sugar in the diet.

Mother of 12-y old boy: "We feel that we have things under control ourselves, but we also feel that our social network sometimes thinks 'didn't they skip something, or haven't they got that wrong?'”

Parents also feared that symptoms of either high or low blood glucose in the child could be interpreted as bad behavior rather than symptoms of a medical condition.

Mother of 6-y old girl: "Sometimes it becomes physical, where she [child with T1D] is just running around the table and sort of tries to get rid of it... Then we have to say, 'now her blood glucose is 15 and that's why she seems misbehaved.' At those times, it is nice to know they [the social network] also have an understanding of what it means to have a blood glucose of 15 ."

Educating the social network: Focus group participants emphasized a great need for the social network of children with T1D to learn about the condition, its consequences, and how to act around the child. Lack of knowledge might affect both safety and the level of social involvement. Parents felt a great responsibility in undertaking this education.

Some parents from the focus group were not comfortable with explaining and educating about T1D, since everything was still new to them. A father of a $6 \mathrm{y} / \mathrm{o}$ girl described it this way:

"We are at a level where we can explain that this is how we do it, but not necessarily explain why we do it."

Parents struggled to pass on a sufficient level of information. One mother explained that she tried to limit the information to the most basic when her daughter was on play-dates. She was afraid that the other parents would be scared and think it was a lot to take on.

Mother of 6-y old girl: "I just explained the most basic things because I didn't want to scare the parents into thinking 'oh no, that's too much to deal with'”

The grandparents and other caregivers described their lack of confidence when it came to treating the child with T1D. One of the main causes of concern around T1D management was diet - and whether high-carbohydrate food should be avoided in general. A common concern among both parents and social network was the risk of hypoglycemia; could the blood glucose suddenly drop and what would happen then?

\section{The value of home education visits}

As described above, family life is deeply affected when a child is diagnosed with T1D and feelings of distress and uncertainty are common in the first period after diagnosis. Thus, the accessibility of support from health care professionals were vital for the families.

Below we elaborate the value of home education visits for each of the key roles comprising the social network: children, parents, siblings, grandparents, friends and their parents.

Parents: After the first month with intense training and struggle to return to a normal life following child's diagnosis; parents described how they had to learn to trust their children in the care of others and they felt a heavy burden in educating their social network about 
T1D management. Hence, one of the most important gains of the home visits for parents was the health professionals taking over the role of educating the network about T1D. Health care professionals represented authority for both parents and social network and they emphasized that it is safe for the children to be in the care of others. This effort, in addition to the informal tone during visits, appeared to enhance parents' trust in their new situation.

The child with T1D and their siblings: Even though the child with T1D was the center of the home education visits, the children rarely participated actively in discussions. In more focus groups, parents described that the children were not paying close attention during the visit. However, the children described the visit as nice, and one boy gave an explanation that might go for most of the childrenthat it was great to have somebody else taking over.

Neither siblings were rarely the center of discussion in the home education visits. In some cases, they did not participate at all. Some parents explained this by not wanting to place a big responsibility on the shoulders of siblings. Yet, reflecting on it during the focus group discussion, some parents realized that involving the siblings in the visits could have been beneficial.

Other parents regretted siblings were not part of the visit, since they perceive older siblings as a great support for the child with T1D.

Grandparents: The families highlighted the important role of the grandparents and that they were an active part of home education visits. Though social roles differed, grandparents were perceived as the closest social relation next to parents, and they often assisted when parents were not at home. This underlines the importance of grandparents being well-informed about T1D management. Grandparents also became frustrated when they experienced conflicting information about T1D from parents, friends and media.

The main point is that the education from the health care professionals created a great deal of knowledge and insight that the grandparents did not possess before the visit. Health care professionals placed great effort on telling the participants that uncertainty about T1D management will not be fatal from one hour to the next. Some of the participating grandparents stressed this as the most important message learned from the health care professionals during home education visits. In addition, more insight about carbohydrate measuring and insulin management was crucial. The families felt that grandparents gained much more confidence in caretaking after participating in the home education visits.

Friends and parents of friends: The participants described a need of informing friends about T1D. This was both to give themselves a sense of safety and to accommodate questions and natural curiosity from friends and classmates. For the children it was important to fit in and follow the social norms, and they saw it was beneficial when somebody else could answer the questions they received and correct any misunderstandings about T1D.

Only one of the five focus groups invited friends and parents of friends to participate in the home education visit. This was the group with the oldest participating child (14 years), and they suggested that the need to inform and educate friends and their parents become more relevant with age. Teenagers are more independent and thus their friends' parents, as well as other social surroundings, can feel unsure of when or whether to interfere.

Father of friend of 14 year old: "I didn't know whether I had to be extra alert when he was visiting my son. Should I check on them regularly in their room? But they [the healthcare professionals] told us we didn't have to, which was great to know."

In focus groups with older children, the potential risks associated with drinking alcohol were a matter of concern. The participants emphasized that friends should be informed about these risks and how to help and support the friend with T1D.

\section{Premises and limitations for the home education visit}

The home as a setting: Focus group participants highlighted advantages of the visits taking place in their home. Parents felt that people from their network were more easily engaged in the visit than if it took place at the hospital. This they explained by less time spent on transport and because they feel safer in a "known" environment.

Both parents and grandparents stressed that when you are at home and feel safe you ask more questions and other types of question, whereas in a hospital setting the feeling of a "clinical" and unfamiliar setting can make you uneasy and stop you from speaking freely.

For the health professionals, meeting the families and their network in the family home was a means to create a more comfortable educational environment and to learn about the life circumstances of the families. Altogether, meeting up in the homely environment has the potential of giving rise to different conversations than what feels natural in a clinical outpatient setting.

Building trust and getting to know the families: Especially grandparents described how meeting the health care professionals' enhanced feelings of trust. They felt assured that they could get assistance from the outpatient clinic whenever needed, which brought a feeling of safety. On the other hand, the health care professionals aimed to learn more about the life of the families, their challenges of living with T1D and how to support children and parents in the outpatient clinic. Though gaining more insight into family life and structures, the health care professionals felt it could still be difficult to assess the social challenges within the families. They worried that there would be needs and challenges in the family that could go unnoticed. Further, the health care professionals expressed concern that the families with the most significant social challenges might be among those declining the offer of the home education visit.

\section{Discussion}

A child being diagnosed with a chronic disease like T1D cause a major impact on family life; their daily life and social ties. Home educations visits targeted families and social network of children with newly diagnosed T1D is a novel approach compared to standard pediatric diabetes care in Denmark. Home education visits educate parents and the child with T1D as well as individuals from the extended family- and social network invited by parents and children with T1D themselves. Further, the educational effort is performed in the home of the family rather than the outpatient clinic. Focus group participants reported that existing prejudices and lack of knowledge among the social network of children with T1D lead to stress and worry for all parties involved. Misunderstandings about T1D 
among the social network produce a sense of fault in the children and their families. Especially the first period following diagnosis is overwhelming for the families, since they are faced with numerous stressors, including the burden of educating their social network. The social network of the families is generally not sure of how to manage T1D and they are afraid of doing something wrong. Subsequently, they might avoid spending time alone with the child. For the health care professionals, the main purpose of home education visits is to reduce fears and create confidence among families and their social network. They teach them to take care of the child with T1D, thereby creating prospects of social support for the family. Among focus group participants, home education visits are perceived to enhance knowledge and the feeling of confidence concerning diabetes care. Thus, there is evidence that home education visits are able to improve knowledge and overcome fear about T1D in the family and social network of the child, and thereby the visits have the potential to support a childhood with reduced restrictions due to T1D.

Literature support the finding that parents of children with T1D are challenged by the various restrictions of daily life, and they are concerned about the future well-being of their children in relation to the social and psychological impact of the disease [8]. Yet, support from social network might serve as a buffer and enhance wellbeing. A study of families with a child diagnosed with congenital heart disease finds that perceived social support is related to positive coping strategies [18]. Accordingly, family strategies of integrating their social network into daily life and care of the child with T1D is crucial.

Yet, as elaborated in the findings of the current study and in keeping with a previous qualitative study from Norway [6], it is a substantial challenge for parents to trust others to take care of their child with T1D. Thus, involving the extended family and social network in educational activities can prove to be an important step towards a more balanced family and social life. For health care professionals in this study, this is the strongest motivation to engage in home education visits. The training performed by the health care professionals excludes any misunderstandings and enhance a feeling of confidence among the participants. In a recent study from Shah and colleagues it is found that different kind of social network is crucial for life wellbeing of adolescents with T1D. They highlight that these finding support the role of health care professionals to provide information about developmentally normative relationships to families and how these can help them respond to childhood T1D [9].

Knowledge on the benefits of home-based diabetes education is sparse. However, a randomized controlled trial from 2016 finds that home-based education on diabetes management proves the same level of patient safety as hospital-based education, and that homebased is the preferred model by a majority of families with a child with T1D [12]. Equally, the current study finds that families and their social network appreciate the benefits of home-based education in terms of convenience and in being in a more relaxed environment.

In this study additionally, the role of siblings became apparent, and some parents expressed they regretted that the siblings did not participate in the home education visits. The parents felt that it was too great a responsibility for siblings to be involved in T1D treatment. Nevertheless, in hindsight they realized that siblings could benefit from taking responsibility and being a good support for the child with T1D. This is in accordance with findings of a recent study reporting that siblings are greatly affected by the T1D diagnosis. They are discontent with the lack of diabetes tasks entrusted with them and often feel overlooked as a resource in the management of T1D [4].

\section{Strengths and Limitations}

The current study displays in-depth insight into the perceptions of homes education visits, with the advantage of exploring a novel method in supporting children with newly diagnosed diabetes and their families.

In terms of recruitment, we engaged a maximum variation strategy, which on the one hand gives broad insight into the major themes of home education visits, but might lack insight into the value for specific roles of the social network. The families were encouraged to invite everyone in their social network, but different relations may have separate needs for information. We found that the family with an adolescent, who invited friends, was interested in more age-related topics i.e. alcohol and partying.

For health care professionals, home education visits, among other things, served as a means to create more insight into the needs of vulnerable families. However, this study cannot conclude whether home education visits are actually successful in reaching vulnerable families. Families with less resources might decline home education visits. Hence, it is a limitation of this study that families who declined home education visits are not included.

\section{Conclusion}

In the first period of time following T1D diagnosis families struggle to overcome the new challenges of living with a chronic disease. Selfmanagement of T1D is challenging. For the parents to hand over care of a child with T1D to e.g. grandparents or parents of playmates reinforce anxiety that something goes wrong. Existing prejudices and lack of knowledge among the social network of children with T1D lead to stress and worry for all parties involved. The social network of the families is generally not sure of how to manage T1D and they are afraid that they will not be able to aid the child with diabetes care. Focus group participants report that home education visits to children with newly diagnosed T1D, their families and social network create sense of security among participants, relieve parents from the burden of educating the network and empower participants from the social network to take care of the child with T1D for shorter or longer duration of time. Hence home education visits has the potential to enhance practical and social support of families with a child with newly diagnosed T1D. We would suggest for more research to focus on how the children with T1D and their siblings could be further engaged in future home education visits. In addition, more research would be needed to unravel potential for home education visits to reach more vulnerable families.

\section{Funding Statement}

The current study is a collaboration between The Department of Pediatrics at Holbaek Hospital and Steno Diabetes Center Zealand. The study received no specific grant from any funding agency in the public, commercial, or not-for-profit sectors.

\section{Ethics and Data Approval}

The study is approved by the Danish "Datatilsyn" (REG-080- 
2019). All data is handled and kept according to General Data Protection Regulation (GDPR).

\section{Patient Consent Statement}

Prior to each interview, written informed consent was obtained from participants above the age of 18 years, while participants under the age of 18 years provided consent through their parents.

\section{Acknowledgements}

Warm thanks to all the children, families and friends participating in these focus group interviews for readily sharing their time and experiences with the research team. Without your participation and honesty, we would not have gained any new insight. Thanks to the Pediatric Ward at Holbæk Hospital, Denmark and the multidisciplinary home visit team for your inspiring work and collaboration in this study. Additionally, thanks to Steno Diabetes Center Sjaelland for making all needed facilities available and kindly supporting this study.

\section{References}

1. Carstensen B, Rønn PF, Jørgensen ME. Prevalence, incidence and mortality of type 1 and type 2 diabetes in Denmark 1996-2016. BMJ Open Diab Res Care. 2020; 8: e001071.

2. Pihoker C, Forsander G, Fantahun B, Virmani A, Corathers S, BenitezAguirre P. ISPAD Clinical Practice Consensus Guidelines 2018: The delivery of ambulatory diabetes care to children and adolescents with diabetes. Pediatric Diabetes. 2018; 19: 84-104.

3. Kingod N, Grabowski D. In a vigilant state of chronic disruption: how parents with a young child with type 1 diabetes negotiate events and moments of uncertainty. Sociology of Health \& Illness. 1. juli 2020;42(6):1473-87.

4. Overgaard M, Lundby-Christensen L, Grabowski D. Disruption, worries and autonomy in the everyday lives of adolescents with type 1 diabetes and their family members: A qualitative study of intrafamilial challenges. Journal of Clinical Nursing. 2020

5. Dybdal D, Tolstrup JS, Sildorf SM, Boisen KA, Svensson J, Skovgaard AM m.fl. Increasing risk of psychiatric morbidity after childhood onset type 1 diabetes: a population-based cohort study. Diabetologia. 2018; 61: 831-838.

6. Iversen AS, Graue M, Haugstvedt A, Råheim M. Being mothers and fathers of a child with type 1 diabetes aged 1 to 7 years: a phenomenological study of parents' experiences. Int J Qual Stud Health Well-being. 2018; 13: 1487758 1487758.
7. Wennick A, Hallström I. Families' lived experience one year after a child was diagnosed with type 1 diabetes. Journal of Advanced Nursing. 2007; 60: 299-307.

8. Fornasini S, Miele F, Piras EM. The Consequences of Type 1 Diabetes Onset on Family Life. An Integrative Review. Journal of Child and Family Studies. 2020; 29: 1467-1483.

9. Shah LL, Ersig AL, Paik A. Social Network Factors and Anxiety among Adolescents with Type 1 Diabetes and Their Parents. J Fam Nurs. 2019; 25: 395-418.

10. Sparud-Lundin C, Hallström I. Parents' Experiences of Two Different Approaches to Diabetes Care in Children Newly Diagnosed With Type 1 Diabetes. Qual Health Res. 2015; 26: 1331-1340.

11. Lowes L, Lyne P, Gregory JW. Childhood diabetes: parents' experience of home management and the first year following diagnosis. Diabetic Medicine. 2004; 21: 531-538.

12. Clapin H, Hop L, Ritchie E, Jayabalan R, Evans M, Browne-Cooper K. Home-based vs inpatient education for children newly diagnosed with type 1 diabetes. Pediatric Diabetes. 2017; 18: 579-587.

13. Clar C, Waugh N, Thomas S. Routine hospital admission versus out-patient or home care in children at diagnosis of type 1 diabetes mellitus. Cochrane Database of Systematic Reviews. 2007.

14. Oduwole A, Uko R, Tade T, Doherty O, Nwodo R, Imafidon E. The effect of home visits on the quality of life of children and adolescents with type 1 diabetes mellitus. Pediatric Diabetes. 2011; 12: 335-340.

15. Tong A, Sainsbury P, Craig J. Consolidated criteria for reporting qualitative research (COREQ): a 32-item checklist for interviews and focus groups. International Journal for Quality in Health Care. 2007; 19: 349-357.

16. Palinkas LA, Horwitz SM, Green CA, Wisdom JP, Duan N, Hoagwood K. Purposeful sampling for qualitative data collection and analysis in mixed method implementation research. Administration and policy in mental health. september 2015; 42: 533-544.

17. Elo S, Kyngäs $H$. The qualitative content analysis process. Journal of Advanced Nursing. 2008; 62: 107-115.

18. Tak YR, McCubbin M. Family stress, perceived social support and coping following the diagnosis of a child's congenital heart disease. Journal of Advanced Nursing. 2002; 39: 190-198. 